\title{
Upregulation of SLAMF3 on human T cells is induced by palmitic acid through the STAT5-PI3K/Akt pathway and features the chronic inflammatory profiles of type 2 diabetes
}

Tong Zhou $\mathbb{0}^{1,2,3,4}$, Guixia Wang ${ }^{2}$, Yanan Lyu ${ }^{1,3,4}$, Lei Wang ${ }^{1,3,4}$, Siyao Zuo ${ }^{1,3,4}$, Jun Zou ${ }^{1,3,4}$, Lin Sun 1,2,3,4, Wenjie Zhao ${ }^{1,3,4}$, Chang Shu', Yong-Guang Yang ${ }^{1,3,4}$ and Zheng Hu (iD)

\begin{abstract}
Metabolic stress-induced low-grade chronic inflammation plays an important role in the development of insulinresistance and type 2 diabetes (T2D). Emerging evidence highlights the importance of directly elucidating T-cell activation under the obesity-induced metabolic stress condition, as $T$ cells primed under such conditions were found to acquire a unique phenotype and function. Herein, we found a significant upregulation of signaling lymphocytic activation molecule family member 3 (SLAMF3) expression on T cells from T2D patients compared to those of healthy controls. Importantly, SLAMF3 upregulation was associated with an increased ability to produce proinflammatory cytokines. Significantly increased SLAMF3 expression was seen in T2D patient T cells that produce IFN- $\gamma$ or IL-17 upon short (4-h) stimulation, compared to non-cytokine-producing T cells. In line with this finding, SLAMF3 high T cells were significantly more sensitive than SLAMF3 ${ }^{\text {low }} T$ cells to TCR stimulation with anti-CD3/CD28 antibodies. Furthermore, treatment with palmitic acid (PA) led to significant upregulation of SLAMF3 on human T cells primed by anti-CD3/CD28 antibodies and on Jurkat cells, a human T-cell line. RNA sequencing revealed strong activation of the PI3K/Akt signaling pathway in T cells that were primed with PA. Further mechanistic studies showed that inhibition of PI3K/Akt signaling, or its upstream mediator STAT5 can prevent PA-induced SLAMF3 upregulation on T cells. These results indicate that SLAMF3 upregulation is associated with T-cell activation and cytokine production in T2D patients, and suggest that elevated saturated fatty acids in T2D patients may induce SLAMF3 upregulation on T cells via activation of the STAT5-PI3K/Akt signaling pathway.
\end{abstract}

Correspondence: Guixia Wang (gwang168@jlu.edu.cn) or

Zheng Hu (zhenghu@jlu.edu.cn)

${ }^{1}$ Key Laboratory of Organ Regeneration \& Transplantation of Ministry of Education, The First Hospital of Jilin University, Changchun 130061, China ${ }^{2}$ Department of Endocrinology and Metabolism, the First Hospital of Jilin University, Changchun 130061, China

Full list of author information is available at the end of the article.

Edited by H.-U. Simon

\section{Introduction}

As a metabolic disorder characterized by insulin resistance and hyperglycemia, type 2 diabetes (T2D) is also associated with low-grade chronic inflammation that may result in a series of diabetes-related complications, including cardiovascular diseases, nephropathy, retinopathy, and increased risk for bacterial infection ${ }^{1}$. T2D patients have been reported to exhibit a decrease in NK, Th2, and regulatory T cells, an increase in Th1 and Th17

\section{(c) The Author(s) 2019}

(c) (i) Open Access This article is licensed under a Creative Commons Attribution 4.0 International License, which permits use, sharing, adaptation, distribution and reproduction in any medium or format, as long as you give appropriate credit to the original author(s) and the source, provide a link to the Creative Commons license, and indicate if changes were made. The images or other third party material in this article are included in the article's Creative Commons license, unless indicated otherwise in a credit line to the material. If material is not included in the article's Creative Commons license and your intended use is not permitted by statutory regulation or exceeds the permitted use, you will need to obtain permission directly from the copyright holder. To view a copy of this license, visit http://creativecommons.org/licenses/by/4.0/. 
cells $^{2,3}$, and a higher production of inflammatory cytokines such as IFN- $\gamma$, IL-17, and TNF- $\alpha^{4}$. Previous studies revealed that $\mathrm{T}$-cell activation and infiltration into obese adipose tissues play an important role in the initiation and progression of adipose tissue inflammation, by recruiting macrophages and inducing obesity-associated insulin resistance ${ }^{5,6}$. In line with these findings, immunotherapy using T-cell-targeted antibody was effective in reversing high-fat diet (HFD)-induced insulin resistance ${ }^{7}$.

An increase in nonesterified fatty acids (NEFAs) is involved in obesity-associated insulin resistance and linked to $\mathrm{T}_{2} \mathrm{DM}^{8}$. It has been reported that palmitic acid (PA), a major component of the saturated NEFAs in HFD, as well as in the plasma of individuals with obesity and T2DM, is a detrimental factor that promotes inflammation and insulin resistance ${ }^{8}$. PA could induce inflammatory injury through an agonist membrane receptor, such as Toll-like receptor 4 (TLR4) ${ }^{9}$, and stimulate T cells to produce reactive oxygen species (ROS) and cytokines ${ }^{10}$. A recent report demonstrated that incubation of mouse CD4 $\mathrm{T}$ cells with PA induces $\mathrm{CD} 4 \mathrm{~T}$ cells to acquire a $\mathrm{CD} 44^{\mathrm{hi}} \mathrm{CCR} 7{ }^{\mathrm{lo}} \mathrm{CD} 62 \mathrm{~L}^{\mathrm{lo}} \mathrm{CXCR} 3{ }^{+} \mathrm{LFA} 1^{+}$proinflammatory phenotype $^{11}$. Although the exact mechanisms are not understood, these reports indicate that PA plays a critical role in inducing inflammatory profiles in T2D.

The signaling lymphocytic activation molecule family member 3 (SLAMF3) is a member of the SLAMF receptors that are widely expressed on lymphohematopoietic cells and regulate immune function through homophilic interactions ${ }^{12}$. $\mathrm{T}$ cells harvested from SLAMF3 ${ }^{-1-}$ mice exhibit poor proliferation and little IL-2 production in response to suboptimal stimulation by anti-CD3 antibody in vitro, indicating that SLAMF3 signaling is involved in T-cell action ${ }^{13}$. Similarly, SLAMF3 has been found to be upregulated in patients with autoimmune disorders, such as systemic lupus erythematosus, supporting its role in promoting T-cell responses ${ }^{14,15}$. However, SLAMF3deficient mice develop spontaneous systemic autoimmunity, suggesting that SLAMF3 may also serve as a receptor contributing to immune tolerance ${ }^{16}$. The reasons for these contradictory observations remain unclear. In this study, we found that SLAMF3 is upregulated on $\mathrm{T}$ cells from $\mathrm{T} 2 \mathrm{D}$ patients. Importantly, increased SLAMF3 expression on $\mathrm{T}$ cells was associated with improved potential to produce proinflammatory cytokines and enhanced responses to TCR stimulation. Further mechanistic studies showed that exposure of $\mathrm{T}$ cells to PA could significantly upregulate SLAMF3 expression through activation of the STAT5-PI3K/Akt signaling pathway, providing direct evidence linking chronic inflammation with elevated plasma NEFAs in T2D patients.

\section{Materials and methods}

\section{Human subjects}

Subjects diagnosed with T2DM $(n=76)$ and healthy control subjects $(\mathrm{HC} ; n=74)$ were recruited from the Department of Endocrinology and Metabolism and the physical examination center of the First Hospital of Jilin University. Exclusions were severe complications (such as renal failure, severe microvascular disease, severe macrovascular disease, or blindness), chronic or common infections (HBV, HCV, influenza, and fever), cancer, longterm use of immunosuppressive agents, or merging with other autoimmune disease. Umbilical cord blood was collected from the Department of Obstetrics of the First Hospital of Jilin University with informed consent for human T-cell function measurement. Protocols involved in the use of human samples were approved by the Institutional Review Board, and all of the experiments were performed in accordance with the protocols.

\section{Flow cytometric assays}

Peripheral blood mononuclear cells (PBMCs) were isolated from heparinized whole blood using density gradient centrifugation from T2D patients or $\mathrm{HCs}$, and stained with various combinations of the following fluorescence conjugated antibodies: anti human-CD3, CD4, CD8, CD25, CD229 (SLAMF3), IL-17, and IFN- $\gamma$ (BD Pharmigen). BD Cytofix/Cytoperm Fixation and Permeabilization Kits (BD) were used for intracellular staining assays to detect cytokine production of $\mathrm{T}$ cells from T2D patients that were stimulated for $4 \mathrm{~h}$ with Leukocyte Activation Cocktail (BD GolgiPlug ${ }^{\mathrm{Tm}}$, including PMA, ionomycin, and brefeldin A). Flow cytometric measurement was performed with a FACS Fortessa (BD Biosciences) and the data were analyzed by FlowJo (version 10).

\section{MACS based cell sorting}

Mononuclear cells were prepared from umbilical cord blood by density gradient centrifugation using Histopaque (1.077, SIGMA), and then sorted for $\mathrm{CD} 4^{+}$cells by MACS positive selection. Briefly, the cells were incubated firstly with biotin-conjugated antihuman CD4 antibody (BD) and then streptavidin-conjugated Micro-beads (Miltenyi Biotec) after washing. $\mathrm{CD} 4^{+} \mathrm{T}$ cells were collected from the cells retained in LS column under magnetic field. The purity of sorted cells was more than $97 \%$ as confirmed by flow cytometric analysis.

\section{Human T-cell expansion assays}

$\mathrm{CD}^{+}{ }^{+} \mathrm{CD} 4^{+} \mathrm{CD} 25^{-}$SLAMF $^{\text {high }} \quad$ (SLAMF3 $^{\text {high }}$ CD 4 $\mathrm{T}$ cells) and $\mathrm{CD} 3^{+} \mathrm{CD} 4^{+} \mathrm{CD} 25^{-}$SLAMF3 $^{\text {low }}$ (SLAMF3 $^{\text {low }}$ $\mathrm{CD} 4 \mathrm{~T}$ cells) were purified by FACS cell sorting (BD Influx) and the purity was confirmed by flow cytometric 
assays ( $>97 \%$ purity). SLAMF3 ${ }^{\text {high }}$ or SLAMF3 ${ }^{\text {low }}$ CD 4 $\mathrm{T}$ cells were incubated in the presence of precoated antihuman CD3 and soluble antihuman CD28 antibodies at the indicated concentrations in $37{ }^{\circ} \mathrm{C}$ incubator with $5 \% \mathrm{CO}_{2}$. Seven days later, the cell expansion was measured by flow cytometric analysis and the images under microscope.

\section{Measuring SLAMF3 expression on human T cells under different conditions}

$\mathrm{CD} 4{ }^{+}$cord blood $\mathrm{T}$ cells were stimulated with CD3/CD28 antibodies (as detailed above) for $72 \mathrm{~h}$ and analyzed for SLAMF3 expression by flow cytometry analysis. We also measured SLAMF3 expression on human $\mathrm{T}$ cell Jurkat cells (pass mycoplasma contamination test) after incubated for $72 \mathrm{~h}$ under similar conditions without addition of antihuman CD3/CD28 antibodies in the absence or presence of PA $(0.1,0.15$, or $0.3 \mathrm{mM})$, PI3K inhibitor (LY294002, sigma), and/or STAT5 inhibitor (sc-355979/Si, SCBT).

\section{RNA sequence}

$\mathrm{CD} 4{ }^{+}$cord blood $\mathrm{T}$ cells were stimulated with $\mathrm{CD} 3 / \mathrm{CD} 28$ antibodies in the absence or presence of $0.3 \mathrm{mM}$ PA for $72 \mathrm{~h}$ in $37^{\circ} \mathrm{C}$ incubator with $5 \% \mathrm{CO}_{2}$. After confirming SLAMF3 expression by flow cytometry analysis, total RNA was extracted from the cells by TRIzol (Invitrogen) and RNA sequencing was performed by Novogene. Differentially expressed genes between CD4 $\mathrm{T}$ cells that were stimulated in the absence or presence of PA were identified using the significance of digital gene expression profiles ${ }^{17}$. Prior to differential gene expression analysis, for each sequenced library, the read counts were adjusted by edgeR program package through one scaling normalized factor. Differential expression analysis of two conditions was performed using the edgeR package ${ }^{18}$. The $p$ values were adjusted using the Benjamini and Hochberg method. Corrected $p$ value of 0.05 and absolute foldchange of two were set as the threshold for significantly differential expression. Kyoto encyclopedia of genes and genomes (KEGG) pathways or Disease Ontology (DO) terms were considered. The method of calculating the $p$ value was performed traditionally ${ }^{19}$. Then, the enriched significance $p$ value was adjusted using the Benjamini and Hochberg algorithm ${ }^{20}$. Finally, KEGG pathways or DO terms with adjusted $p$ values $<0.05$ and including at least two differentially expressed genes were considered.

\section{Statistical analysis}

All analyses were performed with GraphPad Prism version 6. Control and experimental results were compared with the nonparametric Wilcoxon/Kruskal-Wallis or the paired two-tailed Student's $t$-test. For all analysis, statistical significance was reported as follows: ${ }^{*} p<0.05$; ${ }^{* * *} p<0.01 ; * * * 0<0.001 ;$ ns, no significance.

\section{Results \\ SLAMF3 expression is upregulated on T cells from T2D patients}

Seventy-six T2D participants that met the inclusion criteria and 74 healthy controls ( $\mathrm{HCs}$ ) were enrolled in the study (Table 1 ). The mean age and average body mass index (BMI) were 52.15 years and $25.53 \mathrm{~kg} / \mathrm{m}^{2}$ for T2D patients, and 49.37 years and $25.14 \mathrm{~kg} / \mathrm{m}^{2}$ for HCs. T2D patients had an average HbA1c level of $8.81 \%$ and significantly higher fasting blood glucose (FBG; $8.99 \mathrm{mmol} / \mathrm{L})$ than $\mathrm{HCs}(5.23 \pm 0.47 \mathrm{mmol} / \mathrm{L})$. T2D patients also exhibited significantly increased triglyceride (TG; $2.85 \pm 2.41 \mathrm{mmol} / \mathrm{L}$ ) and decreased high-density lipoprotein cholesterol (HDL-C; $1.09 \pm 0.29 \mathrm{mmol} / \mathrm{L}$ ) compared to HCs.

PBMC samples collected from T2D patients and HCs were analyzed for T-cell subsets and phenotypes by flow cytometry. As shown in Fig. S1, T2D patients and HCs had a comparable level of total $\mathrm{CD}^{+}{ }^{+} \mathrm{T}$ cells, but the level of $\mathrm{CD}^{+} \mathrm{CD}^{+} \mathrm{T}$ cells was significantly increased in $\mathrm{T} 2 \mathrm{D}$ patients compared to HCs. Interestingly, a notable change in T2D patients was the upregulated surface expression of SLAMF3 on T cells, including both $\mathrm{CD} 4^{+}$and $\mathrm{CD} 4^{-}$ T-cell subsets (Fig. 1), suggesting a possible involvement of SLAMF3 signaling in altered immune responses in T2D patients.

Table 1 Baseline clinical characteristics and the results of biochemical tests

\begin{tabular}{lll}
\hline & HC & T2DM \\
\hline Total N & 74 & 76 \\
Age & $49.37 \pm 11.34$ & $52.15 \pm 9.11$ \\
Male & 47 & 49 \\
Female & 27 & 27 \\
BMl $\left(\mathrm{kg} / \mathrm{m}^{2}\right)$ & $25.47 \pm 3.72$ & $25.14 \pm 3.42$ \\
FBG $(\mathrm{mmol} / \mathrm{L})$ & $5.23 \pm 0.47$ & $8.99 \pm 2.94^{* * *}$ \\
HbA1c $(\%)$ & - & $8.81 \pm 1.88$ \\
TC $(\mathrm{mmol} / \mathrm{L})$ & $5.15 \pm 0.94$ & $4.82 \pm 1.29$ \\
TG $(\mathrm{mmol} / \mathrm{L})$ & $1.68 \pm 0.98$ & $2.85 \pm 2.41^{* * *}$ \\
LDL-C $(\mathrm{mmol} / \mathrm{L})$ & $3.08 \pm 0.70$ & $2.84 \pm 0.79$ \\
HDL-C $(\mathrm{mmol} / \mathrm{L})$ & $1.47 \pm 0.37$ & $1.09 \pm 0.29^{* * *}$ \\
\hline
\end{tabular}

Results are expressed as the mean \pm SD

$B M I$ body mass index, FBG fasting blood glucose, TC total cholesterol, TG triglyceride, $L D L-C$ low-density lipoprotein cholesterol, $H D L-C$ high-density lipoprotein cholesterol

${ }^{* *} p<0.01,{ }^{* * *} p<0.001$ 


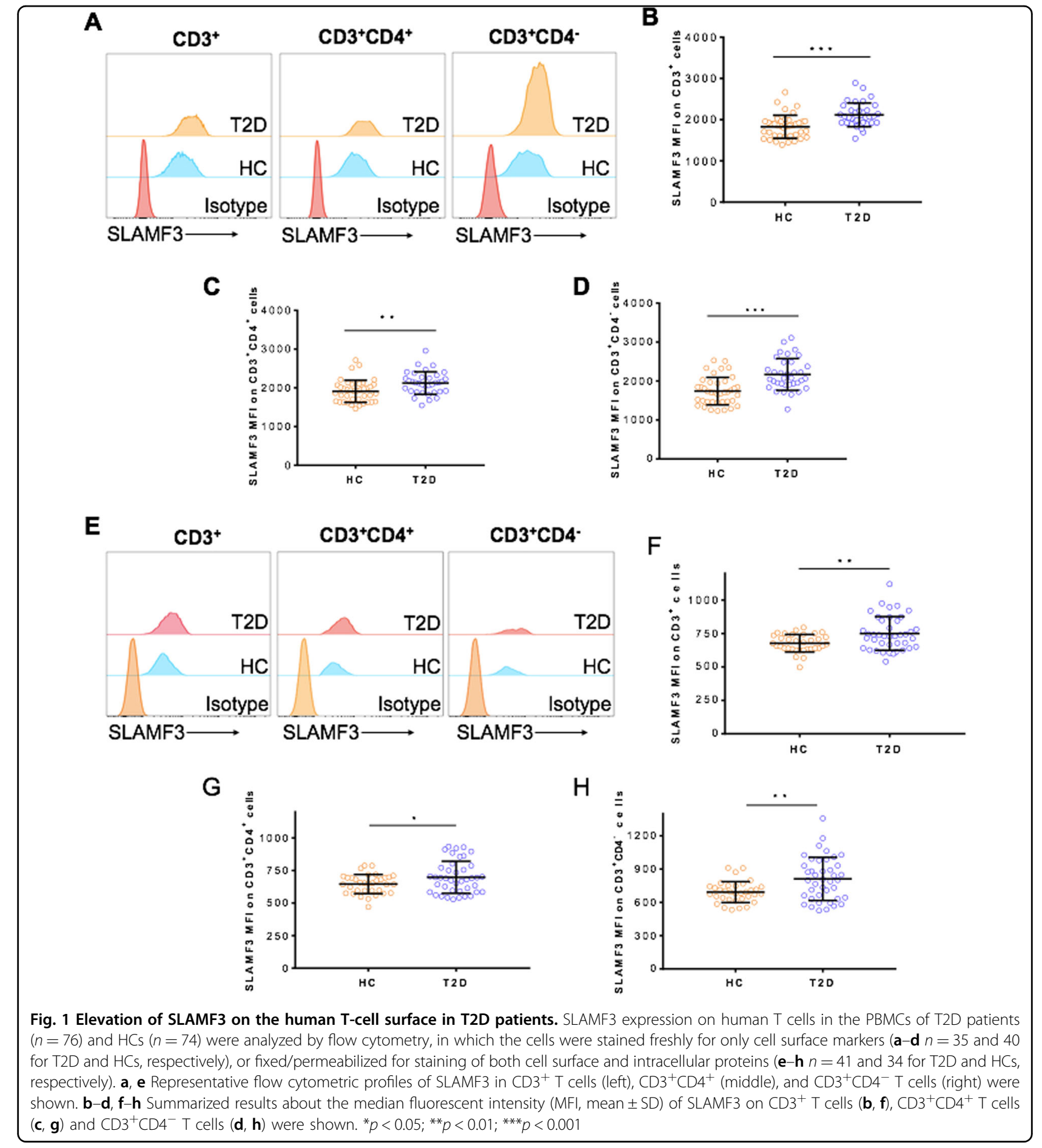

B

Higher surface SLAMF3 expression in T cells is associated with increased proinflammatory cytokine production and improved proliferative responses to anti-CD3/CD28

In T2D patients with chronic low-grade inflammation, a series of proinflammatory cytokines secreted by $\mathrm{T}$ cells (e.g., IFN- $\gamma$ and IL-17) were found at increased levels ${ }^{4}$. Because SLAMF3 has been shown to work as a costimulatory molecule in the activation of human $\mathrm{T}$ cells ${ }^{15}$, we hypothesized that upregulated SLAMF3 expression on $\mathrm{T}$ cells may contribute to the persistent "low-grade inflammatory" status of T2D patients. To address this hypothesis, we compared the levels of SLAMF3 expression on T-cell subsets with different potentials to produce proinflammatory cytokines in T2D 

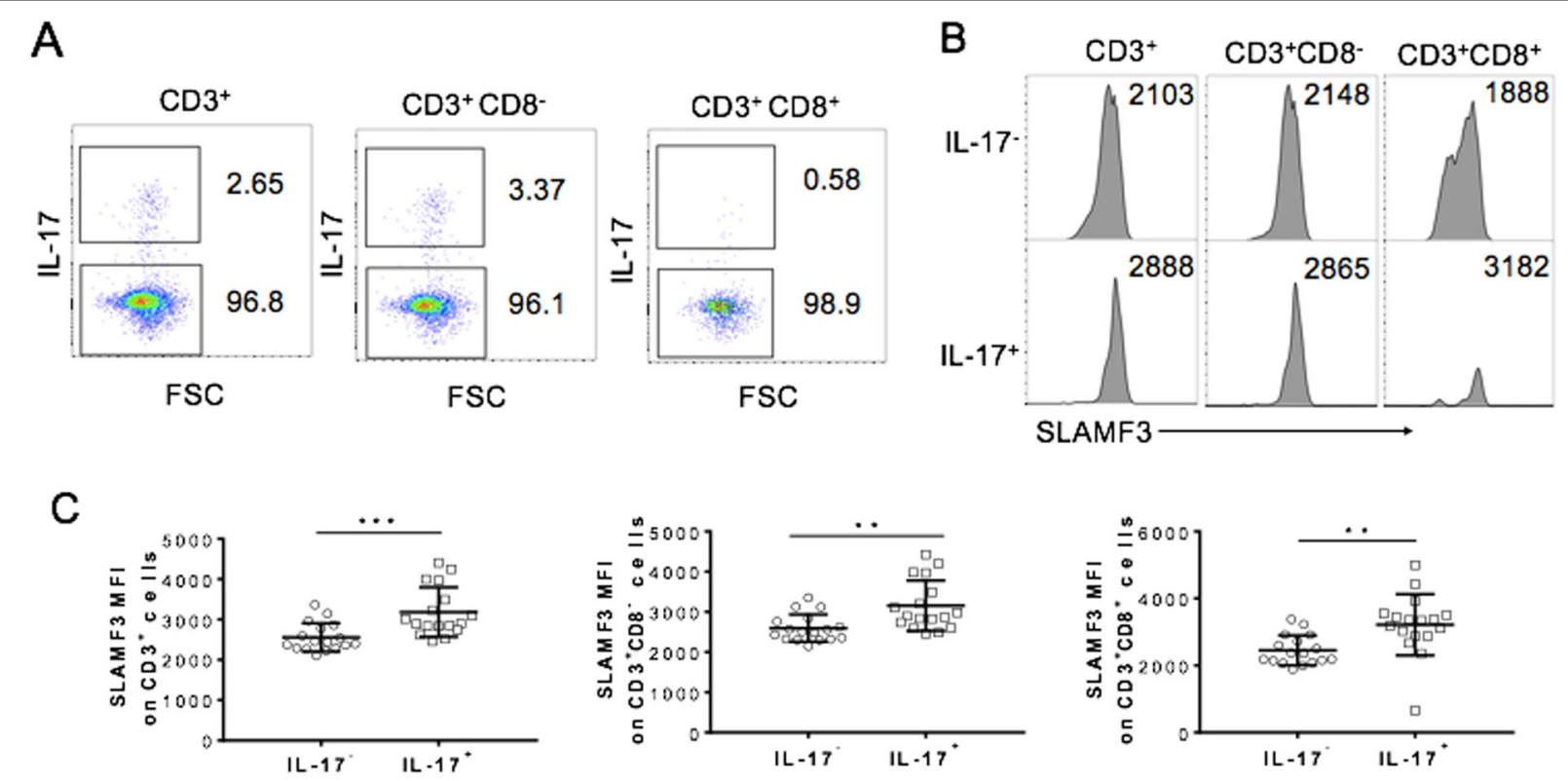

D

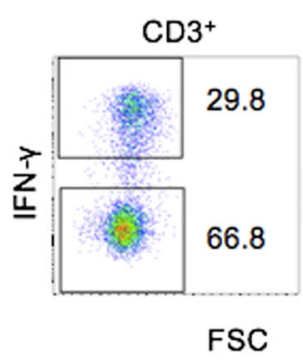

$\mathrm{F}$

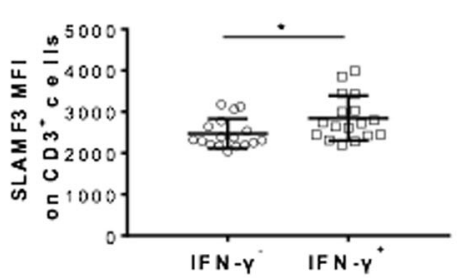

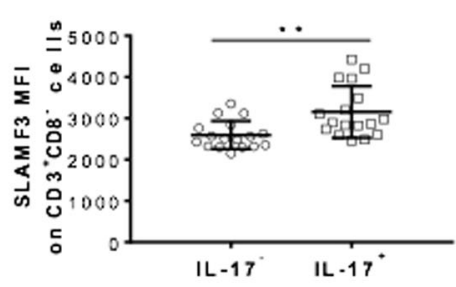
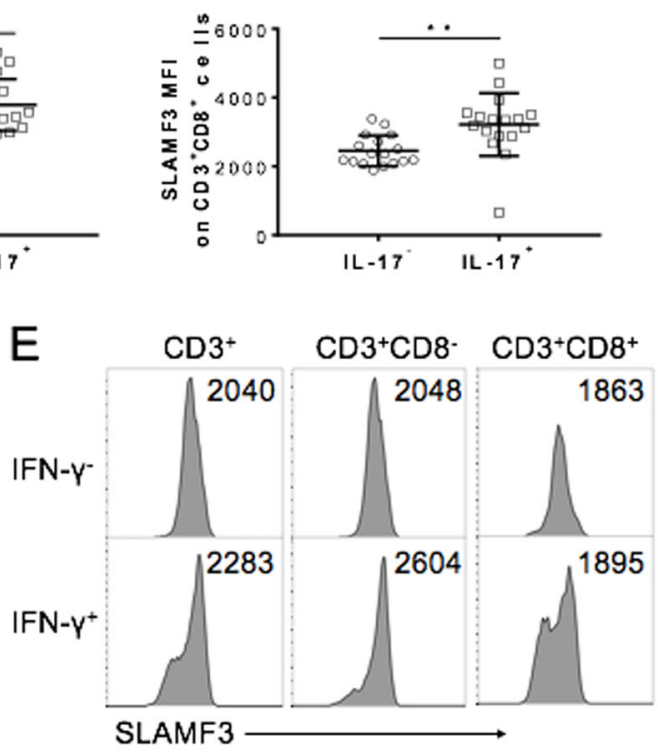

FSC
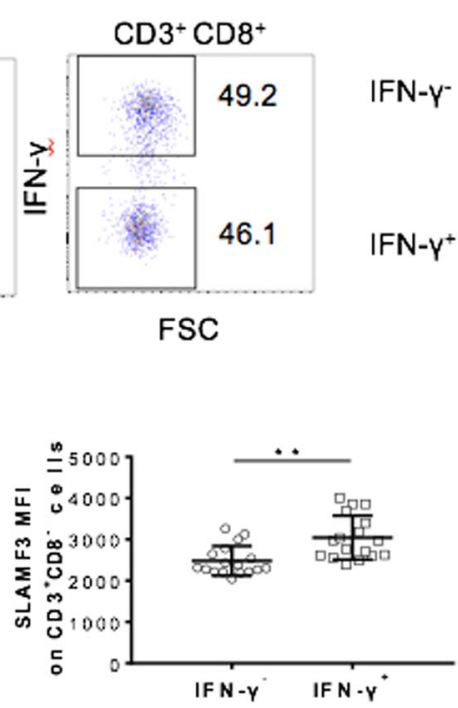

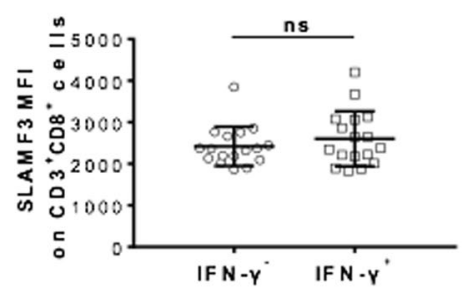

Fig. 2 SLAMF3 ${ }^{\text {high }}$ T cells possess strong capability to produce inflammatory cytokines. PBMCs $(n=17)$ of T2D patients were analyzed for inflammatory cytokine production by flow cytometry after 4-h stimulation by leukocyte activation cocktail. a Representative flow cytometric profiles about production of $\mathrm{IL}-17$ by $\mathrm{CD}_{3}{ }^{+}, \mathrm{CD}^{+}{ }^{+} \mathrm{CD} 8^{-}$, and $\mathrm{CD}^{+} \mathrm{CD}^{+} \mathrm{T}$ cells were shown. $\mathbf{b}$ Representative flow cytometric profiles of SLAMF3 histogram in $\mathrm{IL}-17^{-}$or $\mathrm{IL}-17^{+}$in $\mathrm{CD}^{+}$(left), $\mathrm{CD}^{+} \mathrm{CD}^{-}$(middle), and $\mathrm{CD} 3^{+} \mathrm{CD} 8^{+} \mathrm{T}$ cells (right) were shown. c Summarized data about SLAMF3 MFI analyzed in $\mathrm{IL}-17$ or $\mathrm{IL}-17^{-+}$in $\mathrm{CD}^{+}$(left), $\mathrm{CD}^{+} \mathrm{CD}^{-}$(middle), and $\mathrm{CD}^{+} \mathrm{CD}^{+} \mathrm{T}$ cells (right) were shown. $\mathbf{d}$ Representative flow cytometric profiles about production of IFN- $\gamma$ by $\mathrm{CD}^{+}, \mathrm{CD}^{+} \mathrm{CD}^{-}$, and $\mathrm{CD}^{+} \mathrm{CD}^{+} \mathrm{T}$ cells were shown. e Representative flow cytometric histogram profiles of SLAMF3 analyzed in IFN- $\gamma^{-}$or IFN- $\gamma^{+}$in $\mathrm{CD}^{+}, \mathrm{CD}^{+} \mathrm{CD}^{-}$, and $\mathrm{CD}^{+} \mathrm{CD}^{+} \mathrm{T}$ cells were shown. $\mathbf{f}$ Summarized data about SLAMF3 MFI analyzed in IFN- $\gamma^{-}$or IFN- $\gamma^{+}$in $\mathrm{CD}^{+}, \mathrm{CD}^{+} \mathrm{CD}^{-}$, and $\mathrm{CD}^{+} \mathrm{CD}^{+}$T cells were shown. ns no significant difference; ${ }^{*} p<0.05 ;{ }^{* *} p<0.01 ;{ }^{* * *} p<0.001$

patients. PBMCs from T2D patients were stimulated for $4 \mathrm{~h}$ by PMA/ionomycin with brefeldin $\mathrm{A}$, then $\mathrm{T}$-cell production of IL-17 and IFN- $\gamma$ and expression of SLAMF3 were measured by flow cytometry. Both IL-17and IFN- $\gamma$-producing $\mathrm{CD}^{+} \mathrm{T}$ cells showed significantly increased surface expression of SLAMF3 (Fig. 2). Further analysis revealed that both $\mathrm{CD} 3^{+} \mathrm{CD} 8^{-}$and $\mathrm{CD} 3^{+} \mathrm{CD} 8^{+}$ IL-17-producing cells had significant upregulation of SLAMF3 (Fig. 2a-c). Meanwhile, IFN- $\gamma$-producing $\mathrm{CD}^{+}{ }^{+} \mathrm{CD} 8^{-} \mathrm{T}$ cells but not $\mathrm{CD}^{+}{ }^{+} \mathrm{CD}^{+}{ }^{-} \mathrm{T}$ cells were found with SLAMF3 upregulation (Fig. $2 \mathrm{~d}-\mathrm{f}$ ). These data indicate that higher surface SLAMF3 expression was 

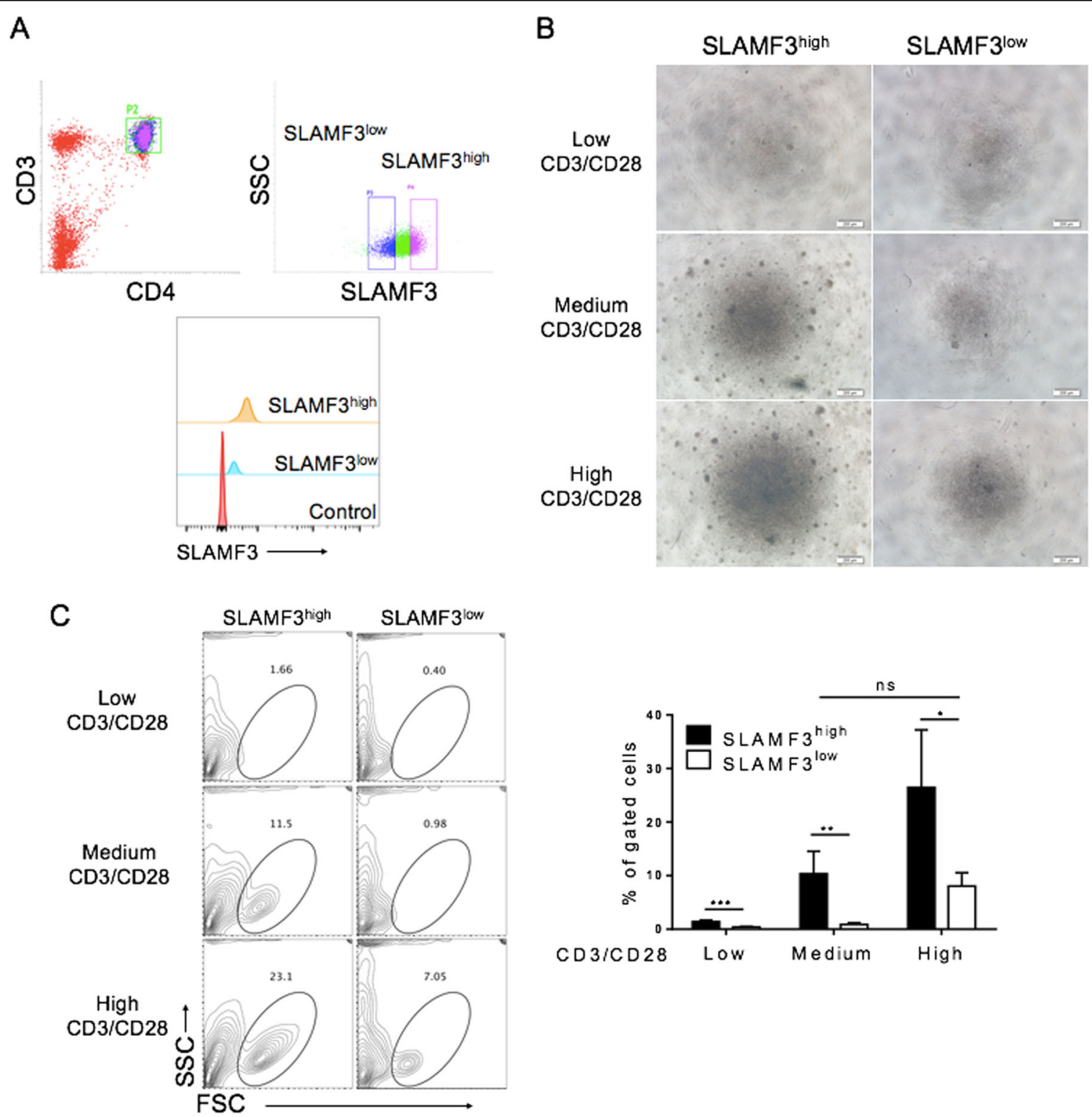

Fig. 3 SLAMF $3^{\text {high }}$ T cells are sensitive to CD3/CD28 stimulation. SLAMF3 ${ }^{\text {high }} \mathrm{CD} 3^{+} \mathrm{CD} 4^{+} \mathrm{CD} 25^{-} \mathrm{T}$ cells and SLAMF3 ${ }^{\text {low }} \mathrm{CD} 3^{+} \mathrm{CD} 4^{+} \mathrm{CD} 25^{-} \mathrm{T}$ cells were purified from cord blood mononuclear cells by FACS sorter, and stimulated with different concentrations of anti-CD3/anti-CD28 antibodies for 7 days in vitro (anti-CD3/anti-CD28 concentration: low $0.8 \mu \mathrm{g} / \mathrm{ml} / 0.4 \mu \mathrm{g} / \mathrm{ml}$, medium $2 \mu \mathrm{g} / \mathrm{ml} / 1 \mu \mathrm{g} / \mathrm{ml}$, and high $5 \mu \mathrm{g} / \mathrm{ml} / 2.5 \mu \mathrm{g} / \mathrm{ml}$ ). a Representative flow cytometric profiles about sorting strategy (up) and the purity of sorted SLAMF $3^{\text {high }}$ or SLAMF3 ${ }^{\text {low }}$ cells were shown. b Representative images of cell expansion 7 days after stimulation. Scale bars equal $200 \mu \mathrm{m}$. c Representative flow cytometric profiles (left) 7 days after stimulation were shown. Summarized data (right) about the ratios of live cells after expansion ( $n=4$ per group, mean \pm SDs) based on flow cytometric analysis was shown. ${ }^{*} p<0.05 ;{ }^{* *} p<0.01 ;{ }^{* * *} p<0.001$. (Data shown are results from a representative of three experiments)

associated with increased IFN- $\gamma$ and IL-17 production in human $\mathrm{T}$ cells from T2D patients.

To further understand the functional differences between $\mathrm{T}$ cells with different levels of SLAMF3 expression, we compared the proliferative response to anti-CD3/ CD28 stimulation of purified SLAMF3 ${ }^{\text {high }}(M F I=677)$ and SLAMF3 $^{\text {low }}(\mathrm{MFI}=318) \quad \mathrm{CD}^{+} \mathrm{CD} 25^{-} \mathrm{T}$ cells (Fig. 3a). SLAMF3 $3^{\text {high }} \mathrm{CD} 4^{+} \mathrm{CD} 25^{-} \mathrm{T}$ cells showed significantly improved proliferation and expansion compared to SLAMF3 ${ }^{\text {low }} \mathrm{CD} 4^{+} \mathrm{CD} 25^{-} \mathrm{T}$ cells at all three concentrations of anti-CD3/CD28 antibodies (referred to as low, medium, and high, respectively; Fig. 3). Robust expansion was only detected in SLAMF3 ${ }^{\text {low }} \mathrm{CD} 4^{+} \mathrm{CD} 25^{-}$ $\mathrm{T}$ cells that were stimulated with the high concentration of anti-CD3/CD28, whereas a comparable expansion was detected in SLAMF3 ${ }^{\text {high }} \mathrm{CD} 4^{+} \mathrm{CD} 25^{-} \mathrm{T}$ cells stimulated 
A

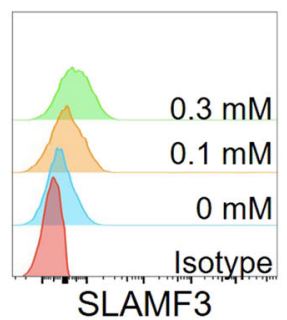

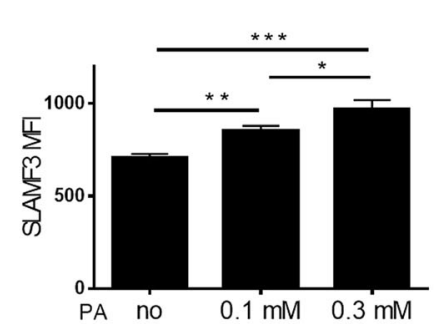

B

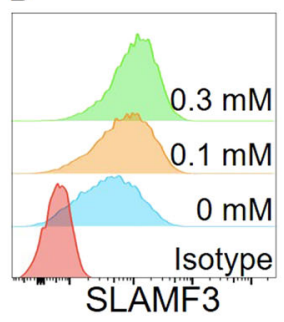

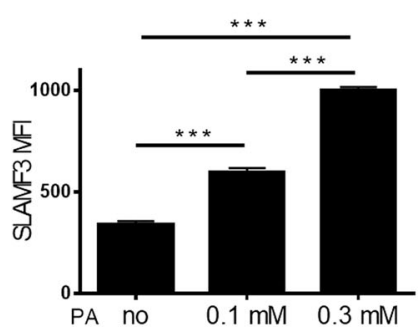

Fig. 4 Palmitic acid treatment raises SLAMF3 on human T cells. a Purified human CD4 T cells were stimulated by $5 \mu \mathrm{g} / \mathrm{ml}$ precoated anti-CD3 and $5 \mu \mathrm{g} / \mathrm{ml}$ solute anti-CD28 antibodies in the presence of palmitic acid $(0,0.1,0.3 \mathrm{mM}, n=3 /$ each) for 3 days. Representative flow cytometric profiles (left) and summarized data (right; mean \pm SDs) about SLAMF3 expression on T cells were shown. (Data shown are results from a representative of four experiments.) b Jurkat cells were cultured in different concentration of palmitic acid (0, 0.1, $0.3 \mathrm{mM}, n=3 /$ each) for 3 days. Representative flow cytometric profiles (left) and summarized data (mean \pm SDs, $n=3 /$ each) about SLAMF3 expression were shown. (Data shown are results from a representative of three experiments)

with the medium concentration of anti-CD3/CD28 (Fig. 3b, c). These data indicate that SLAMF3 ${ }^{\text {high }} \mathrm{T}$ cells are more sensitive to TCR stimulation, providing a possible explanation for the observation that SLAMF3 upregulation was associated with increased potential to produce inflammatory cytokines in $\mathrm{T}$ cells from T2D patients.

\section{Palmitic acids upregulate SLAMF3 expression on human $T$ cells}

As a "bad" fatty acid enriched in HFDs, PAs have been shown to induce T-cell activation and phenotypic alteration $^{5,10}$. Therefore, we considered whether PAs could modulate SLAMF3 expression on human $\mathrm{T}$ cells. We measured SLAMF3 expression on T cells 3 days after stimulation with anti-CD3/CD28, in the absence or presence of PAs. T cells stimulated in the presence of PAs had significantly increased SLAMF3 expression on their surfaces, and the PA effect was dose dependent (Fig. 4a). Similar observations were made in human T-cell line Jurkat cells, in which a significant and dose-dependent upregulation of SLAMF3 expression was detected after culture with PAs, compared to culture without PAs (Fig. 4b).

\section{Palmitic acids upregulate SLAMF3 expression on human $T$ cells through PI3K pathway activation}

To further understand the mechanisms by which PA treatment upregulates SLAMF3 expression on human $\mathrm{T}$ cells, RNA-Seq was performed on purified human $\mathrm{CD}^{+} \mathrm{CD}^{+} \mathrm{T}$ cells that had been stimulated for 3 days with anti-CD3/CD28 without or with PAs $(0.3 \mathrm{mM})$. Differential gene expression analysis revealed that 989 genes were upregulated and 1462 genes were downregulated in PA-treated human T cells (Fig. 5a), which is associated with T2D relevant complications including obesity, pancreas diseases, bacterial infection, coronary artery diseases, and so forth (Fig. S2). Moreover, PA treated human $\mathrm{T}$ cells showed activation of several signaling pathways, particularly those associated with viral infections and cytokine-cytokine receptor interactions (Fig. 5b), which is in agreement with the role of PA in

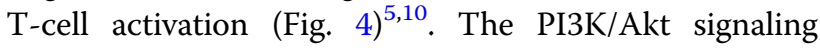
pathway that was also markedly activated in $\mathrm{T}$ cells after PA treatment (Fig. 5b).

In order to verify that the PI3K/Akt signaling pathway activation is involved in SLAMF3 upregulation in PA-treated T cells, we measured SLAMF3 expression on Jurkat cells that were cultured for 3 days in the absence or presence of PAs and/or the PI3K specific inhibitor, LY294002 (10 or $50 \mu \mathrm{M})$. Again, PA treatment significantly upregulated SLAMF3 expression on Jurkat cells, but this SLAMF3 upregulation on PA-treated cells was significantly and dose dependently inhibited by LY294002 (Fig. 6a). The effect of PAs on SLAMF3 expression in Jurkat cells was completely diminished by culture with LY294002 at the higher concentration $50 \mu \mathrm{M}$. Because the JAK/STAT pathway was also activated in PA treated T cells (Fig. 5b) and the JAK/STAT5 signals upstream of PI3K/Akt ${ }^{21-23}$, we measured SLAMF3 expression on Jurkat cells that were cultured with STAT5 inhibitor, sc-355979. As shown in Fig. 6b, culture with sc-355979 also significantly and dose dependently inhibited PA-induced SLAMF3 upregulation, with complete inhibition at $50 \mu \mathrm{g} / \mathrm{ml}$. These data indicate that the STAT5PI3K/Akt signaling pathway is important in mediating PA-induced upregulation of SLAMF3 on human T cells.

\section{Discussion}

Low-grade chronic inflammation is considered a causative factor in the development of insulin resistance and $\mathrm{T}^{2} \mathrm{D}^{1}$. There is emerging evidence for obesity-induced immune responses. Recently, it was reported that memory $\mathrm{T}$ cells from hosts with or without obesity-induced metabolic stress are different in both phenotype and 


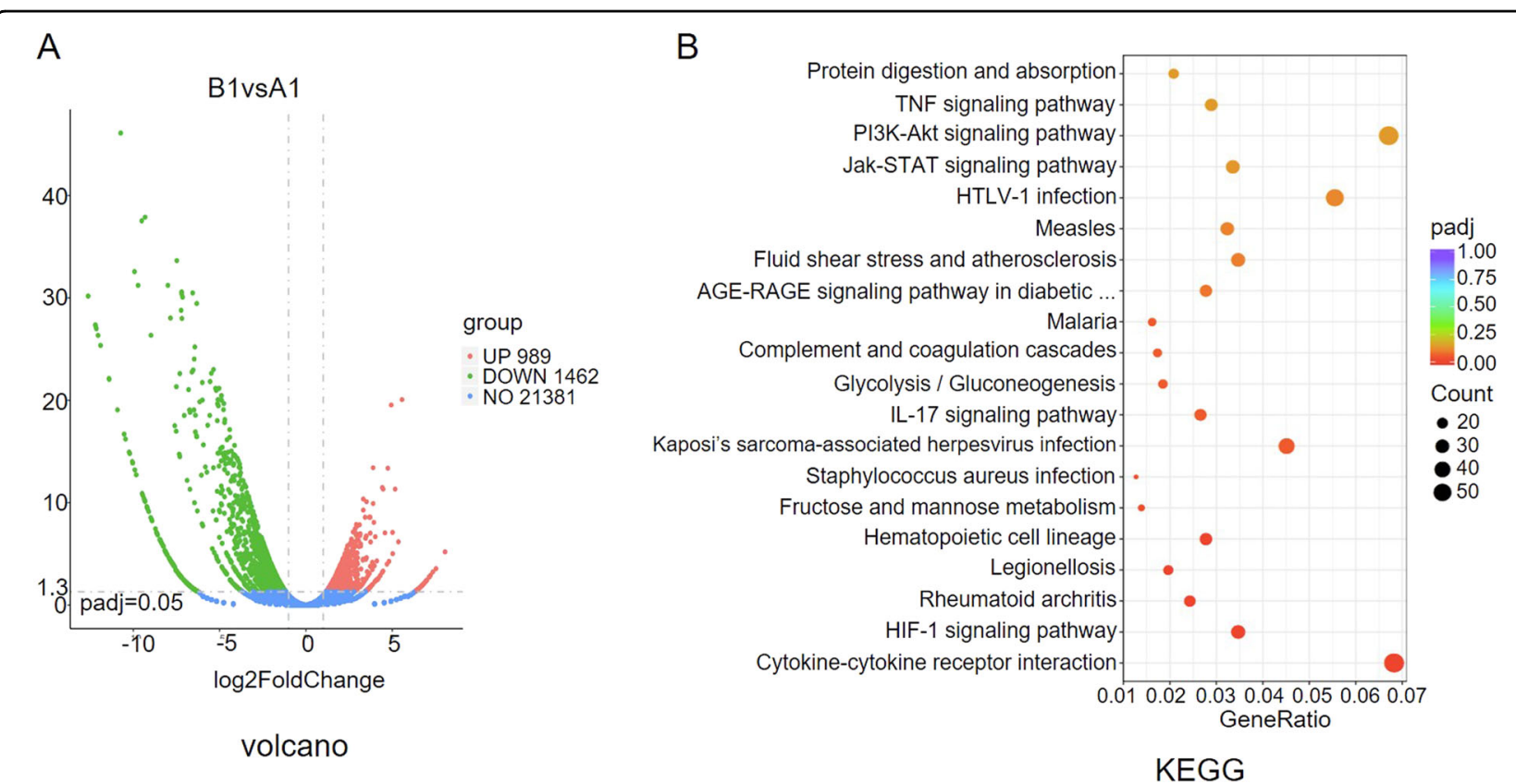

Fig. 5 RNA-Seq analysis of the influences of palmitic acid to human CD4 T cells. Purified human CD4 T cells were stimulated with $5 \mu \mathrm{g} / \mathrm{ml}$ precoated anti-CD3 and $5 \mu \mathrm{g} / \mathrm{ml}$ solute anti-CD28 with or without $0.3 \mathrm{mM}$ palmitic acid for 3 days, the RNA was extracted and send for RNA-Seq. a Volcano profiles of RNA sequence between the two groups. $\mathbf{b}$ Kyoto encyclopedia of genes and genomes (KEGG) pathway analysis of RNA sequence data. ${ }^{*} p<0.05,{ }^{* *} p<0.01,{ }^{* * *} p<0.001$

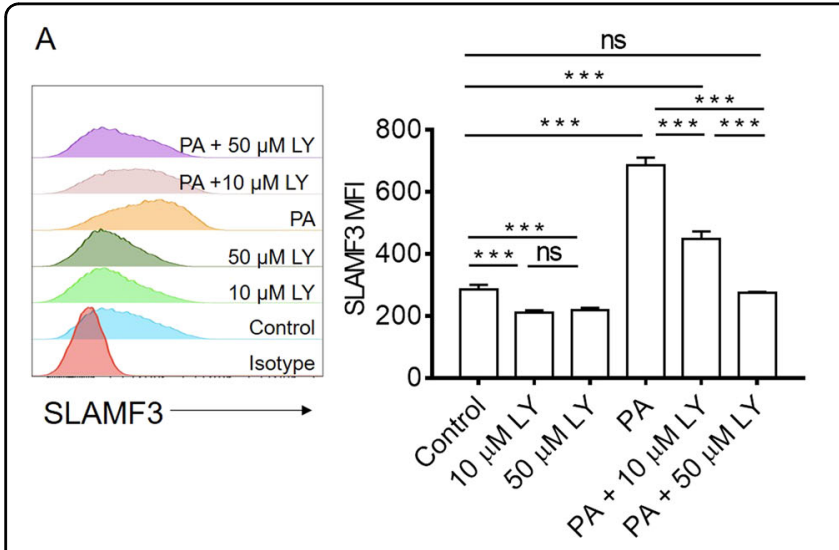

B

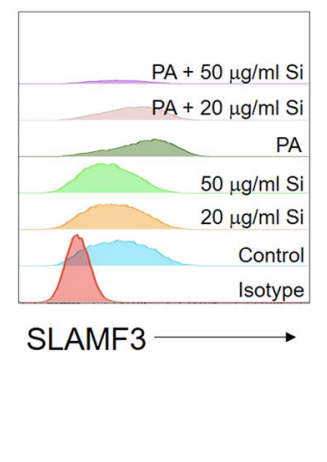

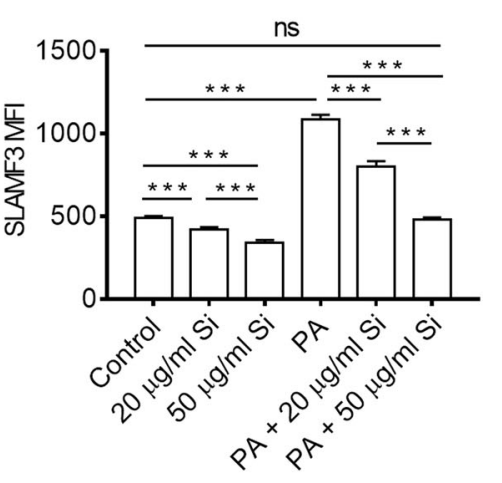

Fig. 6 Inhibition of STAT5-PI3K/Akt pathway prevent the raising of SLAMF3 induced by palmitic acid on human T cells. a Jurkat T cells were cultured in the presence of palmitic acid (PA, 0 or $0.15 \mathrm{mM}$ ) with or without PI3K inhibitor, LY294002 (LY, 10 or $50 \mu M)$ for 3 days. Representative flow cytometric profiles (left) and summarized data (right; mean \pm SDs, $n=4$ /each) about SLAMF3 expression were shown. (Data shown are results from a representative of three experiments.) b Jurkat T cells were cultured in the presence of palmitic acid (PA, 0 or 0.15 mM) with or without STAT5 inhibitor, sc-355979 (Si, 20 or $50 \mathrm{\mu g} / \mathrm{ml}$ ) for 3 days. Representative flow cytometric profiles (left) and summarized data (right; mean \pm SDs, $n=4 / \mathrm{each}$ ) about SLAMF3 expression were shown. (Data shown are results from a representative of three experiments.) ${ }^{* *} p<0.001$; ns no significant difference

function ${ }^{11}$, highlighting the importance of characterizing biased T-cell activation under obesity conditions. Although there are still some controversies, previous studies provided strong evidence supporting the role of SLAMF3 in promoting T-cell activation ${ }^{13-15,24}$. Here we found that SLAMF3 is significantly upregulated on T cells from T2D patients; importantly, its upregulation on
$\mathrm{T}$ cells was associated with increased potential to produce proinflammatory cytokines. In agreement with these observations made in T2D patients, human SLAMF3 ${ }^{\text {high }}$ $\mathrm{T}$ cells were found to be more sensitive to TCR stimulation with anti-CD3/CD28 antibodies. Although our data cannot firmly demonstrate that SLAMF3 upregulation is necessary and/or sufficient for the observed chronic 
inflammatory phenotypes of T cells in T2D patients, these results indicate that SLAMF3 upregulation provides a marker for activated $\mathrm{T}$ cells that are the major source of $\mathrm{T}$ cell-derived proinflammatory cytokines in T2D patients.

PA is a common saturated fatty acid found in the plasma of individuals with obesity and T2DM and plays an important role in the induction of inflammation and the development insulin resistance ${ }^{8}$. PA was reported to interact with agonist membrane receptors (e.g., TLR4) ${ }^{9}$ and to induce ROS and cytokine production ${ }^{10}$. PA can also convert naïve $\mathrm{T}$ cells to a proinflammatory phenotype ${ }^{11}$. These studies raise the possibility that PA is an important factor driving $\mathrm{T}$-cell activation and inflammation in T2D patients. In support of this possibility, we found that PA treatment significantly upregulated SLAMF3 expression on human $\mathrm{T}$ cells through induction of SLAMF3 gene transcripts (Fig. S3A). In addition, PA treatment raised inflammatory gene transcription, e.g., IFNG and IL17A in human T-cell line cells (Fig. S3B and $\mathrm{C}$ ), while not affecting their proliferation (Fig. S4). Moreover, using humanized mice with a functional human immune system ${ }^{25}$, we revealed that HFD feeding induced not only an increase in the visceral adipose tissues, but also an elevation in serum total cholesterol (TC) and triglyceride (TG). Importantly, human $\mathrm{T}$ cells from HFD-treated mice showed a significantly upregulated SLAMF3 expression compared to those from HD-fed mice (Fig. S5). Together, these studies indicate that PA is an important factor causing chronic inflammation in T2D patients, and that PA-induced T-cell activation is associated with the upregulation of SLAMF3 surface expression.

A recent study demonstrated enhanced activation of a PI3K p1108-Akt-dependent pathway in $\mathrm{T}$ cells that were primed in the presence of saturated fatty $\mathrm{acid}^{11}$. The PI3K/Akt pathway is known to be rapidly activated upon T-cell priming and to control transcriptional and metabolic programs that sustain cell activation ${ }^{26}$. In line with these observations, our RNA-Seq results showed that PA induced a markedly enhanced activation of the PI3K/Akt signaling pathway upon priming. Using the pan PI3K inhibitor LY294002, we further confirmed that PI3K/Akt signaling is required for PA-induced SLAMF3 upregulation on anti-CD3/CD28-stimulated $\mathrm{T}$ cells. JAK/STAT5 signals upstream of PI3K/Akt, acting in a linear pathway to modulate $\mathrm{T}$-cell survival and function $^{21-23}$. RNA-Seq data revealed that the JAK/STAT pathway was also activated in PA treated T cells, implying a possible role for STAT5 in driving PI3K/Akt activation in PA-treated $T$ cells. In support of this possibility, we found that PA failed to upregulate SLAMF3 in T cells in the presence of STAT5 inhibitor. Thus, we propose that saturated fatty acid can induce SLAMF3 upregulation on
$\mathrm{T}$ cells through activation of the JAK/STAT5-PI3K/Akt signaling pathway in T2D patients.

In summary, this study shows that SLAMF3 upregulation is associated with increased potential to produce inflammatory cytokines and to respond to TCR signaling of $\mathrm{T}$ cells in T2D patients. Furthermore, saturated fatty acid (e.g., PA) is an important factor that upregulates SLAMF3 expression on $\mathrm{T}$ cells, and this process requires activation of the STAT5-PI3K/Akt signaling pathway. These results provide insight into the immunopathogenesis of T2D and potential molecular targets for T2D therapies.

\section{Acknowledgements}

This work was supported by grants from the Strategic Priority Research Program of the Chinese Academy of Sciences (XDA16030303), Chinese MOST (2015CB964400 and 2017YFA0104402), NSFC (81570145, 81870091, 91642208, 91742107, and 81501279), Plan B for Bethune Project of Jilin University (2015210), an innovation capability project of Jilin Province Development and Reform Commission (2017C019), and an open topic of Jilin Province Science and Technology Agency (20170623092TC-01 and 20180623083TC-01).

\section{Author details}

${ }^{1}$ Key Laboratory of Organ Regeneration \& Transplantation of Ministry of Education, The First Hospital of Jilin University, Changchun 130061, China. ${ }^{2}$ Department of Endocrinology and Metabolism, the First Hospital of Jilin University, Changchun 130061, China. ${ }^{3}$ National-Local Joint Engineering Laboratory of Animal Models for Human Diseases, Changchun 130061, China. ${ }^{4}$ International Center of Future Science, Jilin University, Changchun 130012, China

\section{Author contributions}

T.Z., Y.-N.L., L.W., S.-Y.Z., J.Z., S.L. and C.S. performed the experiments. W.-J.Z. provided the excellent flow cytometric work. Z.T., Y.-G.Y. and Z.H. analyzed the data and wrote the manuscript. G.-X.W. contributed to the development and discussion of the project and edited the manuscript. Z.H. and Y.-G.Y. conceived and designed the study.

Conflict of interest

The authors declare that they have no conflict of interest.

\section{Publisher's note}

Springer Nature remains neutral with regard to jurisdictional claims in published maps and institutional affiliations.

Supplementary Information accompanies this paper at (https://doi.org/ 10.1038/s41419-019-1791-y).

Received: 19 January 2019 Revised: 30 June 2019 Accepted: 4 July 2019 Published online: 22 July 2019

\footnotetext{
References

1. Sell, H., Habich, C. \& Eckel, J. Adaptive immunity in obesity and insulin resistance. Nat. Rev. Endocrinol. 8, 709-716 (2012).

2. Phoksawat, W., Jumnainsong, A., Leelayuwat, N. \& Leelayuwat, C. Aberrant NKG2D expression with IL-17 production of CD4+ T subsets in patients with type 2 diabetes. Immunobiology 222, 944-951 (2017).

3. Cortez-Espinosa, N. et al. CD39 expression on Treg and Th17 cells is associated with metabolic factors in patients with type 2 diabetes. Hum. Immunol. 76, 622-630 (2015)

4. Goldberg, R. B. Cytokine and cytokine-like inflammation markers, endothelial dysfunction, and imbalanced coagulation in development of diabetes and its complications. J. Clin. Endocrinol. Metab. 94, 3171-3182 (2009).
} 
5. Nishimura, S. et al. CD8+ effector T cells contribute to macrophage recruitment and adipose tissue inflammation in obesity. Nat. Med. 15, 914-920 (2009).

6. Kintscher, U. et al. T-lymphocyte infiltration in visceral adipose tissue: a primary event in adipose tissue inflammation and the development of obesitymediated insulin resistance. Arterioscler. Thromb. Vasc. Biol. 28, 1304-1310 (2008).

7. Winer, S. et al. Normalization of obesity-associated insulin resistance through immunotherapy. Nat. Med. 15, 921-929 (2009).

8. Palomer, X., Pizarro-Delgado, J., Barroso, E. \& Vazquez-Carrera, M. Palmitic and oleic acid: the Yin and Yang of fatty acids in Type 2 diabetes mellitus. Trends Endocrinol. Metab. 29, 178-190 (2018).

9. Wang, $Y$. et al. Saturated palmitic acid induces myocardial inflammatory injuries through direct binding to TLR4 accessory protein MD2. Nat. Commun. 8, 13997 (2017).

10. Stentz, F. B. \& Kitabchi, A. E. Palmitic acid-induced activation of human T-lymphocytes and aortic endothelial cells with production of insulin receptors, reactive oxygen species, cytokines, and lipid peroxidation. Biochem. Biophys. Res. Commun. 346, 721-726 (2006).

11. Mauro, C. et al. Obesity-induced metabolic stress leads to biased effector memory CD4(+) T cell differentiation via PI3K p110delta-Akt-mediated signals. Cell Metab. 25, 593-609 (2017).

12. Wu, N. \& Veillette, A. SLAM family receptors in normal immunity and immune pathologies. Curr. Opin. Immunol. 38, 45-51 (2016).

13. Graham, D. B. et al. Ly9 (CD229)-deficient mice exhibit T cell defects yet do not share several phenotypic characteristics associated with SLAM- and SAPdeficient mice. J. Immunol. 176, 291-300 (2006)

14. Chatterjee, M. et al. Increased expression of SLAM receptors SLAMF3 and SLAMF6 in systemic lupus erythematosus T lymphocytes promotes Th17 differentiation. J. Immunol. 188, 1206-1212 (2012).

15. Comte, D. et al. Engagement of SLAMF3 enhances CD4+ T-cell sensitivity to IL-2 and favors regulatory T-cell polarization in systemic lupus erythematosus. Proc. Natl Acad. Sci. USA 113, 9321-9326 (2016).
16. de Salort, J., Cuenca, M., Terhorst, C., Engel, P. \& Romero, X. Ly9 (CD229) cellsurface receptor is crucial for the development of spontaneous autoantibody production to nuclear antigens. Front. Immunol. 4, 225 (2013).

17. Audic, S. \& Claverie, J. M. The significance of digital gene expression profiles. Genome Res. 7, 986-995 (1997).

18. Katz, Y., Wang, E. T., Airoldi, E. M. \& Burge, C. B. Analysis and design of RNA sequencing experiments for identifying isoform regulation. Nat. Methods $\mathbf{7}$, 1009-1015 (2010).

19. $\mathrm{Li}, \mathrm{X}$. et al. RNA-seq analysis of the transcriptome of the liver of cynomolgus monkeys with type 2 diabetes. Gene 651, 118-125 (2018).

20. Benjamini, Y. \& Hochberg, Y. Controlling the false discovery rate: a practical and powerful approach to multiple testing. J. R. Stat. Soc. Ser. B. Stat. Method. 57, 289-300 (1995)

21. Hand, T. W. et al. Differential effects of STAT5 and PI3K/AKT signaling on effector and memory CD8 T-cell survival. Proc. Natl Acad. Sci. USA 107 16601-16606 (2010).

22. Radler, P. D., Wehde, B. L. \& Wagner, K. U. Crosstalk between STAT5 activation and PI3KJAKT functions in normal and transformed mammary epithelial cells. Mol. Cell. Endocrinol. 451, 31-39 (2017).

23. Wofford, J. A., Wieman, H. L., Jacobs, S. R., Zhao, Y. \& Rathmell, J. C. IL-7 promotes Glut1 trafficking and glucose uptake via STAT5-mediated activation of Akt to support T-cell survival. Blood 111, 2101-2111 (2008).

24. Chehimi, M., Vidal, H. \& Eljaafari, A. Pathogenic role of IL-17-producing immune cells in obesity, and related inflammatory diseases. J. Clin. Med. 6, 1-19 (2017)

25. Lan, P., Tonomura, N., Shimizu, A., Wang, S. \& Yang, Y. G. Reconstitution of a functional human immune system in immunodeficient mice through combined human fetal thymus/liver and CD34+ cell transplantation. Blood $\mathbf{1 0 8}$ 487-492 (2006)

26. Essig, K. et al. Roquin suppresses the PI3K-mTOR signaling pathway to inhibit T helper cell differentiation and conversion of treg to Tfr cells. Immunity $\mathbf{4 7}$ 1067-1082 e1012 (2017). 